\title{
Transition Region Blinkers
}

\author{
C. E. Parnell ${ }^{1}$, D. Bewsher ${ }^{1}$, R. A. Harrison ${ }^{2,1}$ and A. W. Hood ${ }^{1}$ \\ 1 School of Mathematical and Computational Sciences, University of St \\ Andrews, North Haugh, St Andrews, Fife, KY16 9SS, SCOTLAND \\ 2 Rutherford Appleton Laboratory, Chilton, Didcot, Oxon, OX11 0QX, \\ ENGLAND
}

\begin{abstract}
To allow an objective study of transition region blinkers an automated identification program has been developed to analyse the CDS/SoHO data. The general properties of blinkers identified by this method confirmed the properties of those identified by eye in previous studies. Blinkers have typical lifetimes of about 20 minutes, areas of about $2.8 \times 10^{7} \mathrm{~km}^{2}$ and intensity enhancement factors of about 2 . For the first time, blinkers have been identified in active regions. These blinkers have the same general properties as quiet-region blinkers. The automated approach found many more blinkers than previously had been found giving a global birth rates of about $9 \mathrm{~s}^{-1}$ for quiet-region blinkers and 16 $\mathrm{s}^{-1}$ for active-region blinkers. Most blinkers, active or quiet region, are red shifted with typical velocities of about $13 \mathrm{~km} \mathrm{~s}^{-1}$. Finally, we can confirm that blinkers are not enhancements in temperature, but either enhancements in density or increases in the filling factor.
\end{abstract}

\section{Introduction}

Small bright intensity enhancements, named blinkers, were observed in the Transition Region by Harrison et al. $(1997 ; 1999)$ using the CDS/SoHO. They are most readily identified in $\mathrm{O} \mathrm{V}$, but can also be found in other EUV lines, such as, $\mathrm{O}$ IV, O III, He I and He II. Blinkers have been found in quiet regions and typically last between 1 and 40 minutes (average 17 minutes), have areas of between $2-5 \times 10^{7} \mathrm{~km}^{2}$ and intensity enhancement factors of 1.5 for the $\mathrm{O} \mathrm{V}$ and $\mathrm{O}$ IV lines and 1.3 for O III. Ratios of the O V, O IV and O III intensity light curves of blinkers are found to be flat. This implies that the intensity enhancements seen in blinkers are not due to temperature, but rather due to an increase in density or filling factor. Harrison et al. (1997) found the global birth rate of blinkers to be just $1 \mathrm{~s}^{-1}$. However, blinkers have also been identified using He II images taken by EIT/SoHO (Berghmans et al., 1998). Here their global birth rate was determined to be between $20-40 \mathrm{~s}^{-1}$.

This initial work on blinkers reveals some interesting features, but there are still many questions to be answered. For instance, do blinkers only occur in quiet regions or can they also occur in active regions and coronal holes? Do blinkers have coherent plasma motions, and if so what are they? What type of 
magnetic field configurations lie below blinkers in the Photosphere? We have tried to answer these questions in this paper.

\section{Data: CDS and MDI}

Data from two CDS runs has been analysed. These data sets both have a slit size of roughly 4" x 240 " and a raster area of 41 " x 124 " containing $10 \times 73$ pixels sized $4 " \times 1.6 "$. The exposure time is 10 seconds making a cadence of 2.5 minutes for each raster. Each data set contains rasters taken simultaneously in six different lines. He I $(584.33 \AA)$, formed at $2 \times 10^{4} \mathrm{~K}$, is the only Chromospheric line. O III (599.59 $\AA)$ O IV $(554.52 \AA)$ and O V $(629.73 \AA)$ are all transition region lines and are formed at $10^{5} \mathrm{~K}, 1.6 \times 10^{5} \mathrm{~K}$ and $2.5 \times 10^{5} \mathrm{~K}$, respectively, and the two coronal lines, $\mathrm{Mg} \operatorname{IX}(368.06 \AA)$ and $\mathrm{Mg} \mathrm{X}(624.94 \AA)$ are formed, respectively, at $10^{6} \mathrm{~K}$ and $1.2 \times 10^{6} \mathrm{~K}$. To determine the connection of blinkers to the photospheric magnetic field high resolution partial frame images from MDI/SoHO are used. These images have a spatial resolution of $0.6 " \mathrm{x} 0.6$ ", and a temporal resolution of 1 minute.

We have designed an automated identification program to objectively study blinkers. The essential ingredients of the program are:

- to prep the data and compensate for rotation

- to estimate a global average error, $\sigma$

- to identify collections of 3 or more pixels that have simultaneous temporal peaks that are greater than $n \sigma$ above the background intensity, where $n$ is an integer (each such collection is called a blinker)

\section{Results}

The S15251 data set, taken on 29th November 1998, was centred in a quiet region near disk centre. During the 11 hrs 45 mins observing sequence 161 blinkers were identified with peaks of $5 \sigma$ or greater. The typical properties of the blinkers detected are given in the table below (Table 1). The second data set, S11478, was again near disk centre, but this time it lay over a large sunspot in an active region. It was taken on 18th June 1998 and lasted just 2 hrs 50 mins. Blinkers were discovered to also occur in active regions with 72 being observed in this data set. The typical properties of active-region blinkers are very similar to those of quiet-region blinkers (Table 1). Furthermore, the typical lifetimes, areas and intensity factors observed agreed well with previous estimates suggesting that the automated indentification program worked well. It has been found that the rise and fall phases of the blinker are very similar in length and so blinkers do not appear to have a flare like intensity profile.

The global birth rates for quiet-region and active-region blinkers have been estimated at $9 \mathrm{~s}^{-1}$ and $16 \mathrm{~s}^{-1}$, respectively. These rates are considerably higher than the rate quoted by Harrison et al. (1997). The reason for this increase is the much better and more efficient detection procedure we have employed.

The ratios of the oxygen ion light curves are also investigated. These are found to be effectively flat and so confirm previous results that suggests that 


\begin{tabular}{lcccc}
\hline O V Properties & Quiet Region: $5 \sigma$ & \multicolumn{2}{c}{ Active Region: $5 \sigma$} \\
\hline \hline Number of blinkers & \multicolumn{2}{c}{161} & \multicolumn{2}{c}{72} \\
Global birth rate $\left(\mathrm{s}^{-1}\right)$ & \multicolumn{2}{c}{9} & \multicolumn{2}{c}{16} \\
Lifetime (mins) & $5-87$ & $($ av 22) & $5-68$ & (av 20) \\
Area $\left(\times 10^{7} \mathrm{~km}^{2}\right)$ & $1.0-13.9$ & $($ av 2.7) & $1.0-10.7$ & (av 3.4) \\
Intensity enhancement factor & $1.4-4.3$ & (av 1.9) & $1.2-8.3$ & (av 2.4) \\
Rise time (mins) & $2.5-37$ & (av 10) & $2.5-29$ & (av 9) \\
Fall time (mins) & $2.5-55$ & $($ av 11) & $2.5-44$ & (av 11) \\
& & & & \\
Doppler shift & Red & Blue & Red & Blue \\
Numbers with $\left|v_{b u l k}\right|>3 \mathrm{~km} \mathrm{~s}^{-1}$ & 82 & 32 & 45 & 10 \\
Mean $\left|v_{\text {bulk }}\right|\left(\mathrm{km} \mathrm{s}^{-1}\right)$ & 11.6 & -6.8 & 14.8 & -8.5 \\
\hline
\end{tabular}

Table 1. Typical properties of blinkers observed in $\mathrm{O} \mathrm{V}$

blinkers are due to increases in density or filling factor as opposed to temperature.

At the time of peak emission from the blinker, spectral data was analysed to look for line-of-sight motions in the blinker. The results (Table 1) suggest that the majority of blinkers are red shifted by about $13 \mathrm{~km} \mathrm{~s}^{-1}$.

Finally, the blinkers were compared with MDI observations of the magnetic flux below. All the quiet-region blinkers were observed in or near network regions which had strong converging motions and relatively strong concentrations of flux. At one of these regions a cancelling magnetic feature is observed above which a bright point is visible in the Mg IX and Mg X lines. About half of the blinkers observed occur below this bright point. For the active-region blinkers a few were found around the edge of the sunspot in regions where out flowing flux interacted with the surrounding opposite polarity field and a significant number of blinkers were observed directly above the sunspot. This may suggest that blinkers occur near sites of strong converging flow and it is these flows, rather than the cancellation that regularly occurs at these sites that is important.

\section{References}

Berghmans, D., Clette, F. and Moses, D. 1998, A\&A, 336, 1039-1055

Harrison, R.A. 1997, Solar Physics, 175, 467-485

Harrison, R.A., Lang, J., Brooks, D.H. and Innes, D.E. 1999, A\&A, 351, 11151132 